\title{
The combined monitoring of brain stem auditory evoked potentials and intracranial pressure in coma. A study of 57 patients
}

Luis García-Larrea, François Artru, Olivier Bertrand, Jacques Pernier, François Mauguière

\begin{abstract}
Continuous monitoring of brainstem auditory evoked potentials (BAEPs) was carried out in 57 comatose patients for periods ranging from 5 hours to 13 days. In 53 cases intracranial pressure (ICP) was also simultaneously monitored. The study of relative changes of evoked potentials over time proved more relevant to prognosis than the mere consideration of "statistical normality" of waveforms; thus progressive degradation of the BAEPs was associated with a bad outcome even if the responses remained within normal limits. Contrary to previous reports, a normal BAEP obtained during the second week of coma did not necessarily indicate a good vital outcome; it could, however, do so in cases with a low probability of secondary insults. The simultaneous study of BAEPs and ICP showed that apparently significant ( $>40 \mathrm{~mm} \mathrm{Hg}$ ) acute rises in ICP were not always followed by BAEP changes. The stability of BAEP's despite "significant" ICP rises was associated in our patients with a high probability of survival, while prolongation of central latency of BAEPs in response to ICP modifications was almost invariably followed by brain death. Continuous monitoring of brainstem responses provided a useful physiological counterpart to physical parameters such as ICP. Serial recording of cortical EPs should be added to BAEP monitoring to permit the early detection of rostrocaudal deterioration.
\end{abstract}

$(尹$ Neurol Neurosurg Psychiatry 1992;55:792-798)

EEG Department, Hôpital Neurologique, Lyon

L García-Larrea

F Mauguière

Anaesthesiology

Department, Hôpital

Neurologique, Lyon

F Artru

INSERM U280, Lyon,

France

O Bertrand

J Pernier

Correspondence to:

Dr Garcia-Larrea, CERMEP

(Hôpital Neurologique), 59

Bvd Pinel, 69003 Lyon,

France

Received 17 May 1991 and in final revised form 18 December 1991 . Accepted 19 December Accep
EP has been recorded. Furthermore, slow but steady degradations of EPs, with a time course of several hours or days, have every chance of being unnoticed if a single recording session takes place when latencies and amplitudes, though progressively degrading, still remain within normal standard limits.

In previous reports on BAEP monitoring we described the irreversible, preterminal BAEP changes preceding brain death, ${ }^{713}$ as well as some drastic but reversible abnormalities associated with the use of sedative drugs. ${ }^{8}$ We now report data from high-rate monitoring in a series of 57 deeply comatose patients, with special emphasis on the significance of transient and reversible BAEP changes related to acute fluctuations of the intracranial (ICP) or cerebral perfusion (CPP) pressures. This study also addresses the question of the prognostic significance of single BAEPs recorded at different moments of the patient's evolution.

\section{Patients and methods}

\section{a) BAEP monitoring system}

The monitoring system used for this study has been described in detail elsewhere. ${ }^{5}$ Briefly, it consists of a one-channel standard EP device coupled to a microcomputer (IBM-PC) which controls all operations. The recording electrodes are either $\mathbf{A g} / \mathrm{AgCl}$ disks fixed with collodion jelly or sterilised subcutaneous needles placed at $\mathrm{Cz}$ (positive input of the preamplifier) and over the mastoid process ipsilateral to the stimulated ear (negative input). A third electrode over the contralateral mastoid process serves as ground. The stimuli, delivered at a rate of $20 / \mathrm{s}$ through an ear-inserted transducer, are non-filtered alternating clicks of $\mathbf{8 0}$ or $90 \mathrm{~dB} \mathrm{HL}$ (the intensity being kept constant during a monitoring session). BAEPs are analog filtered with a bandpass of $150-1300 \mathrm{~Hz}$. Then an adaptive digital filter based on Wiener's filtering theory is calculated automatically and readapted by the computer for each new BAEP. ${ }^{514}$ This adaptive filter usually obtains interpretable traces after 200 to 400 stimulations (fig 1), thus reducing the recording time to $10-20 \mathrm{~s}$ for each individual BAEP.

At the beginning of the monitoring session 5 to 10 standard BAEPs were recorded to check the reliability and reproducibility of the responses. Waves I, III and V were identified by the examiner on these first records and their latencies, calculated by means of a cursor, served as reference values for subsequent automatic peak detection. The rate of BAEP 

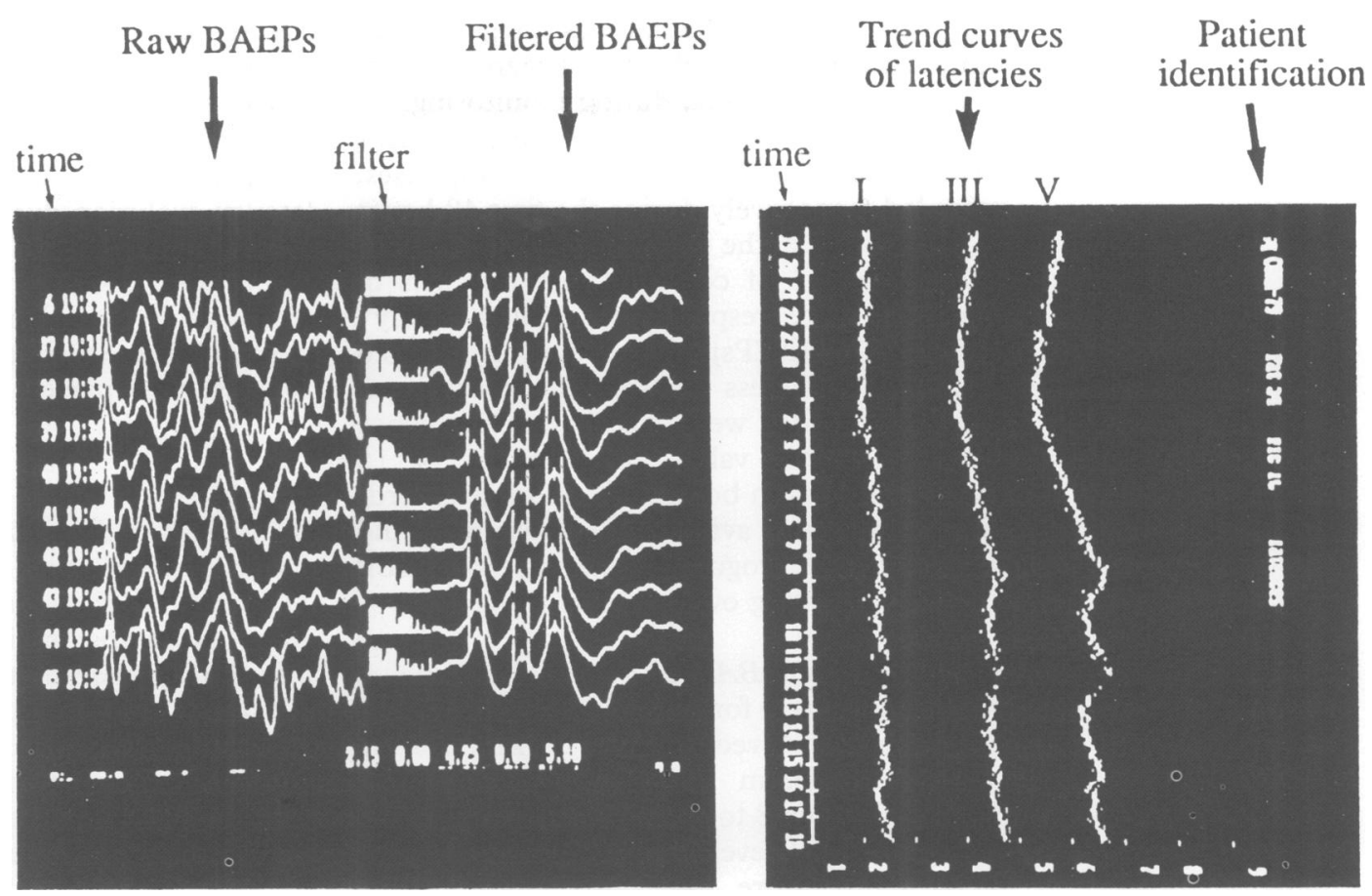

Figure 1 Display of sequential BAEP traces and trend curves of latencies in a comatose patient. BAEP's to 400 click stimuli were obtained once every 2 minutes. Raw traces (left column) were filtered by means of an adaptive digital filter that changed for every new recording; the corresponding filtered BAEPs appear in the middle column. Waves I, III and $V$ on filtered BAEPs were automatically detected by the computer within a "latency window" (cursors) which was recalculated automatically after each new recording (see Methods). The right part of the figure illustrates latency trends of waves I, III and V during the last 24 hours of monitoring. The time is represented on a vertical scale, the bottom 10 points corresponding to the 10 traces illustrated on the left part of the figure. The progressive latency increases of wave $V$ between 2-9 am is non-pathological in origin and corresponds to the combined intravenous infusion of lidocain and sodium thiopental.

recording was usually of 1 new EP every 3-5 minutes, but could be modified at any moment during monitoring. The last 10 responses obtained, as well as the trend curves of latencies corresponding to the last 24 hours of monitoring, were permanently displayed on the computer screen at the patient's bedside (fig 1). BAEP recording, adaptive digital filtering, automatic peak detection, disk storage and screen display were usually performed in less than 1 minute. Intracranial pressure (ICP) values were also digitised and displayed on the screen along with BAEPs

\section{b) Patients}

Fifty seven patients, all of them deeply comatose (Glasgow Coma Scale $\leqslant 6$ in 54 cases, $<8$ in the others) were monitored during periods ranging from 5 hours to 13 days. The mean (SD) duration of monitoring was 98 (50) hours, that is about four days; the mean number of BAEP recordings obtained per patient was 1920 (range: 40-7000). The aetiology of coma was supratentorial in 55 patients $(97 \%)$, while a primary brainstem lesion could be demonstrated in the remaining two. The origin of coma was traumatic in 35 cases (61\%) and vascular in $22(39 \%)$. The patients' mean (SD) age was 35 (16) (range 6-64). As expected, the traumatic group was significantly younger, mean (SD) years $=27(13)$ than the vascular group, 46 (14) years. Metabolic or anoxic comas were not included in the study; our conclusions therefore only concern coma of traumatic or vascular orgin.

Clinical outcome was evaluated at three months. Death occurred in 29 cases $(50 \cdot 8 \%)$ as a direct result of the brain lesions. Three patients $(5 \cdot 3 \%)$ developed a persistent vegetative state (PVS), of whom one died two years later. Finally, 4 patients $(7 \%)$ died from non neurological causes apparently unrelated to the aetiology of coma (generalised sepsis in 2 and cardiac failure in the other 2). The rate of unfavourable outcome (death or PVS) was significantly greater within the stroke group than in head injured subjects $(77 \%$ vs $53 \%$ respectively, $p<0.05)$.

In 53 patients ( $93 \%$ of the series) ICP and arterial pressure were also continuously monitored. ICP values were obtained by means of an epidural sensor ${ }^{15}$ and stored on floppy disks along with the neurophysiological parameters. Cerebral perfusion pressure (CPP) was computed as the difference between mean arterial and intracranial pressures (MAP-ICP). Due to technical mishaps the ICP values were considered unreliable in 2 cases by the intensive care practitioner (FA); consequently correlations with BAEP could only be attempted in 51 patients.

\section{c) Normative data and abnormality criteria Single BAEPs}

Normative data were obtained from 40 control subjects with normal hearing. ${ }^{16}$ For each response the I-V interpeak latency (IPL), the V/I amplitude ratio and the amplitude of waves III and V were assessed, with normal limits set at the mean value $\pm 3 \mathrm{SD}$ of our control group. Due to possible injury to the peripheral auditory system in traumatic coma, the absence of 
any reproducible potential was considered as significant only if preceded by a progressive loss of components verified during monitoring.

The prognostic implications of single BAEPs were studied in two different sets of responses, recorded respectively during the first 48 hours of coma and at the beginning of the second week (day 6-7 of coma). These two sets of responses were respectively labelled "early" and "late" BAEPs; they were investigated separately to assess whether the moment at which recordings were made could influence their prognostic value. In a subset of 15 patients data from both, early and late recording sessions were available. In them we could investigate the prognostic relevance of BAEP changes occurring over the first week.

\section{Dynamic study of BAEPs}

Normative data for the dynamic study of BAEPs were derived from 10 normal hearing subjects in whom auditory responses were recorded during 8 to 10 consecutive hours at a rate of 1 BAEP every 10 minutes. EEG and rectal temperature were also monitored in these subjects to assess the physiological variations of responses related to body temperature and vigilance status. A total of 590 BAEPs were obtained [mean (SD) 58 (10) per subject], including recordings during physiological sleep in all subjects. BAEP latencies during monitoring were best characterised by their mean (SD) value, this latter providing a criterion of latency stability. In our control subjects $S D$ was $0.15 \mathrm{~ms}$ for wave $V$ latency, and $0.1 \mathrm{~ms}$ for I-V IPL.

Transient modifications of the I-V interval detected during monitoring were considered significant when exceeding $2.5 \mathrm{SD}$, on the condition that at least 10 consecutive BAEPs were available during the increase and decrease of latency, so that a regression line could be calculated for the successive latency values in each phase.

BAEP modifications related to body temperature changes or observed under blood levels of anaesthetic drugs known to alter BAEPs ${ }^{17}$ were excluded, after previous studies in which the effects of such non-pathological factors on BAEP monitoring were systematically assessed. ${ }^{5781318}$

\section{ICP and CPP changes}

Acute pressure modifications were considered significant on the two conditions: 1) that ICP

Table 1 Comparison (unpaired $t$ test) of several variables within the groups of "early" $(n=27)$ and "late" $(n=26)$ normal BAEPs. In patients with "early" normal responses (left half of the table) neither the age nor the interpeak latencies (I-V IPL) could discriminate between survivors and non-survivors. Conversely, in patients with "late" normal BAEPs the I-V interval was significantly longer in those who subsequently died. Values in the two lower rows are mean (SD). Age in years, I-V IPL in milliseconds).

\begin{tabular}{|c|c|c|c|c|c|c|}
\hline & \multicolumn{3}{|c|}{$\begin{array}{l}\text { "Early" Normal BAEPs } \\
\text { (first } 24 \text { hours) }(n=27)\end{array}$} & \multicolumn{3}{|c|}{$\begin{array}{l}\text { "Late Normal BAEPs } \\
\text { (6th-7th day) }(n=26)\end{array}$} \\
\hline & Survival & & Death & Survival & & Death \\
\hline $\begin{array}{l}\text { N (\%) } \\
\text { Age } \\
\text { I-V IPL }\end{array}$ & $\begin{array}{c}13(48 \cdot 1) \\
33(17) \\
3.97(0.3)\end{array}$ & $\begin{array}{l}\text { NS } \\
\text { NS } \\
\text { NS }\end{array}$ & $\begin{array}{c}14(51 \cdot 1) \\
39(16) \\
4.03(0.3)\end{array}$ & $\begin{array}{l}13(50) \\
30(17) \\
4.04(0.3)\end{array}$ & $\begin{array}{l}\text { NS } \\
\text { NS }\end{array}$ & $\begin{array}{c}13(50) \\
31(17) \\
4.26(0.2) \\
\mathrm{p}<0.05\end{array}$ \\
\hline
\end{tabular}

raised beyond $40 \mathrm{mmHg}$ or CPP decreased below this same level, and 2) that the episode lasted a minimum of 10 minutes. On these criteria we recorded pressure abnormalities in 26 patients of the series $(42 \cdot 3 \%)$; BAEP latency evolution during these episodes could be reliably studied in all but four of them, in whom the brainstem responses were either abolished (one patient) or altered by high doses of drugs (three cases) at the moment that ICP rise took place.

\section{Results}

$A$ "Static" study of BAEPs during coma.

Prognostic implications

1) Initial BAEP and life outcome

After excluding patients under continuous infusion of anaesthetic agents which altered significantly the responses, the relationship between "early" BAEPs (obtained during the first 48 hours of coma) and clinical outcome was studied in 37 patients.

Twenty seven patients had initially normal BAEPs and $14(51.8 \%)$ recovered full consciousness, while in 13 cases $(48 \cdot 1 \%)$ the evolution was towards death. Within this group of normal BAEPs, neither the mean age, nor the I-V interval at monitoring onset could discriminate between patients with good and bad outcome (table 1).

BAEPs were initially abnormal in 10 patients. Nine of these had supratentorial hemispheric lesions, who all died. The remaining patient had an isolated haemorrhagic brainstem contusion that could explain BAEP changes, and recovered from coma.

\section{2) "Late" BAEPs and outcome}

"Late" BAEPs (obtained during the second week of coma) were recorded in 30 patients. Four of them had abnormal or absent responses, all of who died. Of 26 patients with

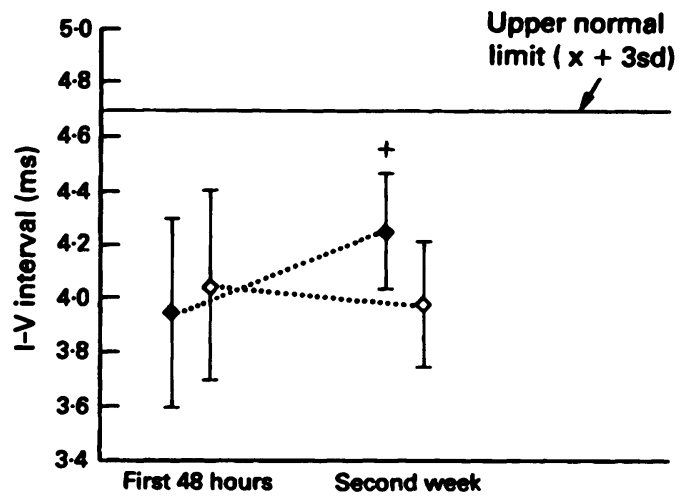

Figure 2 Temporal evolution of the I-V interval (IPL) in 15 patients continuously monitored during the first 8-10 days of coma. Values from patients who survived (open circles) and from those who subsequently died (filled circles) are presented separately. In the survivors group the I-V IPL remained stable between the first 48 hours and the seventh day of coma (open circles), while this measure increased significantly in the group of patients who sebsequently died (filled circles, the cross means $p<0.05$ on paired $t$ test). Clinical outcome was assessed at three months. Note that, despite the changes operated between the two recording sessions, the interpeak latencies remained within normal limits (mean $\pm 3 S D$ ) in both groups. 

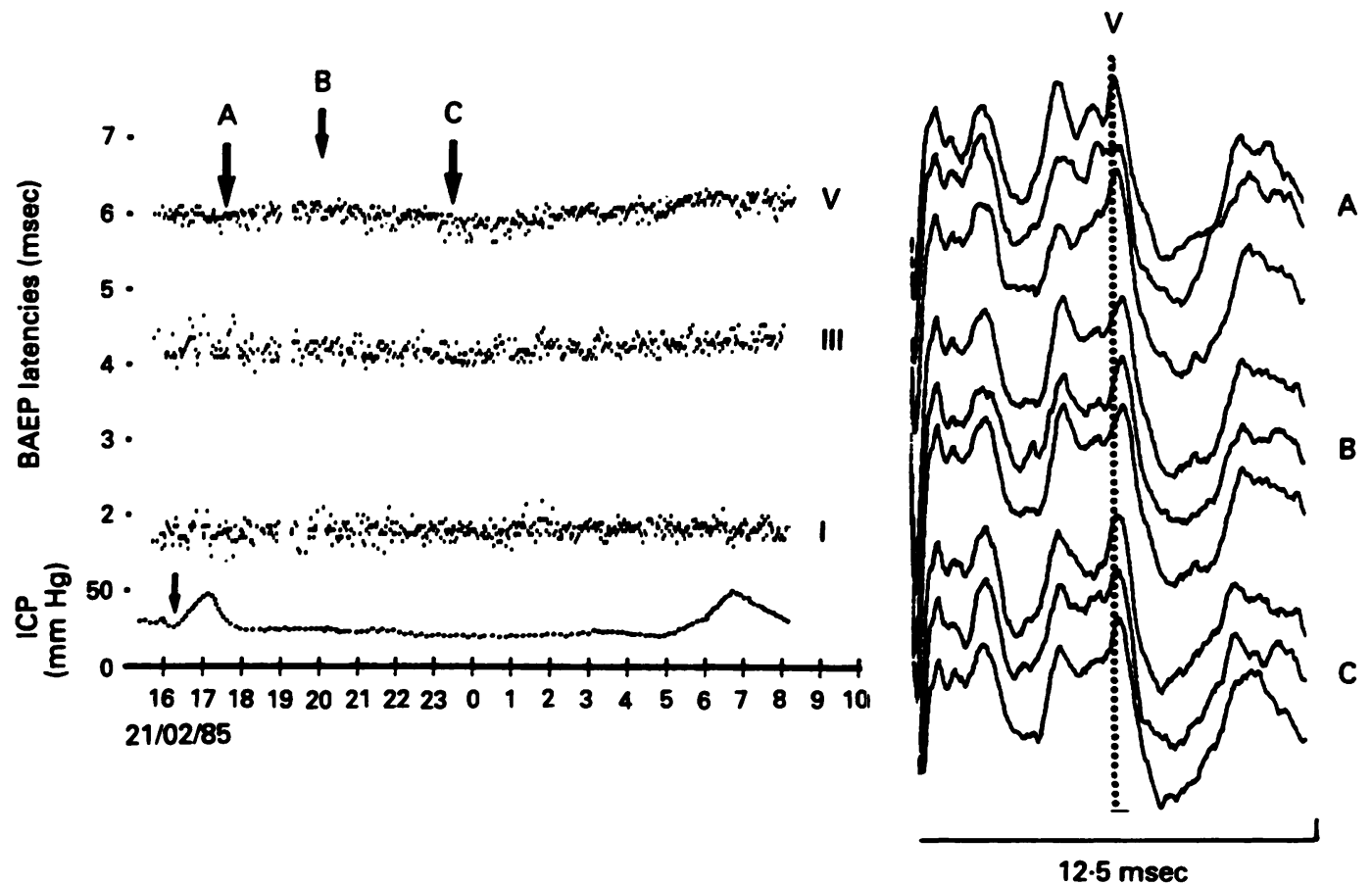

Figure 3 Long-lasting, transient latency increase during coma monitoring. The left part of the figure shows the trend curves of BAEP latencies (three upper graphs) and of intracranial pressure values (bottom line). The onset of the episode of BAEP latency increase $(A)$ coincided with an acute rise of ICP (arrow on bottom trace) but lasted several hours longer. Letters $A, B$ and $C$ mark corresponding periods of monitoring in trend curves (left) and BAEP traces (right). Thus, traces on the right are representative $B A E P$ s obtained at the onset $(A)$, the moment of maximal latency increase $(B)$ and the end of the episode (C). Each illustrated trace corresponds to the average of 5 consecutive BAEPs (1000 stim, 10 minutes of monitoring). Note that, in spite of the significant effect seen on trend curves the actual increase of wave $V$ latency on BAEP traces was very small. After the end of the episode a slow latency increase developed in the absence of new ICP changes, but was enhanced when a new ICP rise appeared at 6 am. BAEP monitoring was discontinued at 10 am, when BAEPs were still present; the patient died six hours later.

normal "late" BAEPs 13 survived and 13 died (4 of them from presumed non-neurological causes). Thus a normal BAEP recorded beyond the first days of coma did not warrant a good outcome in our patients. However, the $\mathrm{I}-\mathrm{V}$ interval at one week's evolution was significantly shorter in the patients who survived than in those who died (unpaired $t$ test, $\mathrm{p}<0.05$, see table 1 ), although it remained within normal limits in both cases. The mean age was comparable in the first and in the second group.

When data from the "early" (first 48 hours) and "late" (second week) recording sessions were compared no significant modification of the I-V IPL was observed in the survivors, whereas patients who subsequently died showed a significant increase of this parameter (paired $t$ test $\mathrm{p}<0.05$, fig 2).

Brain death occurred in only 2 of the 9 patients $(22 \%)$ with either no visible lesions on CT scans, or only extracerebral lesions such as epi- or subdural haematomas, and who showed normal 'late' BAEPs. Conversely, death occurred in 11 of 17 patients $(65 \%)$ with normal BAEPs on the second week of coma and intracerebal haemorrhagic lesions (cerebral haemorrhages or haemorrhagic infarcts)

\section{B) Dynamic study of BAEP changes during coma \\ 1) Transient $B A E P$ changes}

Applying the criteria specified in the methods section, 25 episodes of transient, reversible latency changes of the I-V IPL were observed in 18 patients of our series (figs 3 and 4). Despite such latency increase the I-V IPL remained within normal standard limits $(<4.7 \mathrm{~ms})$ in all except one patient. The duration of IPL variation episodes ranged from two to 12 hours. Only interpeak latencies were taken into account since amplitudes were in our experience too susceptible to uncontrolled sources of variations to be fully reliable.

The overall mortality in this group was significantly higher than that observed in the patients who did not present transient abnormalities of the I-V IPL (14/18 (78\%) vs $16 / 33$ $(48.5 \%), p<0.05)$. This difference was still increased if only patients with normal BAEPs at monitoring onset were considered, in which case the mortality associated with transient IPL increases was of $76 \%$, in contrast with $37 \%$ for all other patients. The mean (SD) age of patients in both groups was not significantly different, [34.6 (17) vs 35.6 (16) years] and could not account for the difference in outcome.

\section{2) Acute ICP/CPP modifications associated to} $B A E P$ changes

As shown in table 2, patients with acute increases of the I-V interval were more likely to have ICP/CPP modifications during monitoring than those without BAEP changes. Conversely, the existence of significant episodes of pressure modification was not associated with a higher proportion of BAEP abnormalities. 




Figure 4 Short-lasting ICP and BAEP changes during monitoring. This patient presented repetitive acute rises of intracranial pressure of 1-2 hours duration lower trace), accompanied in each case by a corresponding increase in wave V latency (upper trend curve) with stability of earlier waves I and III. One of such episodes is illustrated by the figure, its onset being marked by the big vertical arrows. Note the almost exact coincidence of pressure rise and latency increase. A subsequent rise of ICP, beginning at 22 hours, also entailed a latency increase of wave $V$ (right small arrows). Unfortunately $B A E P$ monitoring had to be interrupted due to a technical problem, and the descending limb of BAEP and ICP trend curves could not be recorded.

Only in half of the patients were pressure changes followed by acute BAEP latency increase; in the others, BAEP remained stable during and after the episode of ICP/CPP alterations (table 2).

Reversible increases of the I-V interval were time-locked to the ICP/CPP changes in 11 patients, of whom $10(90.9 \%)$ had an unfavourable outcome towards cerebral death. In 11 other patients BAEP latencies remained unchanged in spite of acute pressure modifications. Nine of them ( $82 \%)$ survived. From the two patients who died one had very abnormal BAEPs from the beginning of monitoring and the remaining died because of an intercurrent sepsis. In the remaining four patients BAEP evolution during acute ICP changes could not be assessed, either due to the high doses of sedative drugs which prevented BAEP interpretation (three cases), or because of BAEPs being already abolished at the time of ICP rise (one case). Thus the association of acute ICP/ CPP modification plus I-V increase was followed by neurological death in $91 \%$ of cases, whereas mortality was only $18 \%$ when acute ICP/CPP changes were not accompanied by transient BAEP latency increase. The difference is highly significant $(p<0.001)$.
Table 2 Distribution of the 57 patients monitored according to the existence of a) transient modifications of intracranial or cerebral perfusion pressures, and b) transient increases of the I-V interpeak latency. The existence of latency increase was very frequently (11/15) accompanied by pressure changes; however, pressure modifications were only followed by a BAEP latency increase in $50 \%$ of cases. As discussed in the text, an unfavourable outcome was associated to the concomitant occurrence of $B A E P$ and pressure changes

\begin{tabular}{llrrl}
\hline & & \multicolumn{3}{l}{ ICP/CPP modifications } \\
\cline { 3 - 5 } & & Yes & No & $N D^{*}$ \\
\hline I-V IPL increase & Yes & 11 & 4 & 3 \\
& No & 11 & 20 & 2 \\
& NDt & 4 & 1 & 1 \\
\hline
\end{tabular}

"ICP non controlled.

+BAEP absent or unreliable due to high levels of lidocaine/ thiopental.

ND = Not done.

\section{Discussion}

A) Prognostic implications of single $B A E P$ recordings at different moments of the evolution Gross abnormalities or abolition of BAEPs in a comatose patient carry an ominous prognosis, provided that lesions to the peripheral auditory system have been ruled out. ${ }^{19-31}$ The poor prognosis associated with this pattern during coma is illustrated by the $90 \%$ mortality observed in our group of patients with abnormal BAEPs at monitoring onset.

The central latency of BAEPs has been reported as being almost constantly normal when recorded during the first six hours of traumatic coma. ${ }^{30}$ This suggests that most abnormalities encountered later are secondary to hemisphere lesions, and reflect an ongoing process of rostrocaudal deterioration of the brainstem. Discrete brainstem lesions may, however, also induce severe BAEP abnormalities if they involve the central auditory pathways. ${ }^{33}$ BAEP abnormalities in those cases reflect the primary brainstem lesion, and not the rostrocaudal deterioration of brainstem function, and the ensuing outcome is not necessarily bad. This was the case of one of our patients with severely abnormal BAEPs resulting from a small pontine haemorrhage, without evidence of hemispheric lesion, who recovered from coma some weeks later.

Contrary to what happens with abnormal brainstem responses, normal BAEPs during the first days of coma do not bring much relevant information on the final outcome. In published series the average incidence of unfavourable evolution (severe disabilities, PVS or death) in patients with bilaterally normal BAEPs during the acute phase of traumatic coma is about $30 \%$, but ranges from 0 to more than $50 \%$. $^{3134}$ In our study $46 \%$ of patients with normal "early" BAEPs did unfavourably, a figure comparable to other series dealing with patients having low Glasgow Coma Scores. ${ }^{24} 25$

Some studies have suggested that a favourable vital outcome might be predicted by normal BAEPs recorded beyond the first days of coma. ${ }^{192027}$ The reason for this is probably that death after traumatic or vascular coma mainly occurs during the first week, and especially during the first 48 hours after the 
accident. $^{35-37}$ We addressed this question by investigating 26 patients of our series who had normal "late" BAEPs recorded during the second week of coma, 15 of whom had also been studied within the first 48 hours.

When the group of 26 patients is considered as a whole, "late" normal BAEPs do not prove of greater prognostic value than "early" normal responses, since mortality was still $50 \%$ (13 patients). However, the nature of the lesion might also influence the prognostic value of normal "late" BAEPs, since death occurred in only two of nine cases (22\%) without focal injury on CT scans, or with extracerebral lesions not associated to intraparenchimal involvement, whereas brain death ensued in 11 of 17 patients $(65 \%)$ who had intracerebral haemorrhagic lesions, in spite of persisting normal BAEP's during the second week. Thus only in a small subset of patients may normal "late" BAEPs be associated with a good vital outcome. This subgroup did not include patients with intracerebral haemorrhages or haemorrhagic infarcts, which is not surprising since such lesions have the highest potentiality for late neurological deterioration. ${ }^{36}$ As a general rule, the smaller the probability of a secondary insult (such as, late ICP rise, rebleeding) the better the prognostic value of a "late" normal BAEP.

The expected outcome may be optimised by assessing the dynamics of brainstem responses, rather than their statistical normality at any time of coma evolution. One of the main reasons contributing to the lack of prognostic power of normal BAEPs is that "normal" waveforms are in many cases progressively deteriorating responses that will remain within normal limits for a long time. This point is illustrated by fig 2 , which shows central latency changes in our patients between the first and second weeks of coma. Although latencies increased significantly in the group of patients who subsequently died, all values remained within statistical normal limits in both recording sessions, this stresses the limitations of statistical "normality' when applied to comatose patients.

\section{B) Transient $B A E P$ changes during coma}

Transient BAEP changes lasting from minutes to hours are frequent in comatose patients. Many of these are non-pathological in origin and mainly related to temperature or drug levels modifications; however, detection of clinically relevant episodes is crucial, for they may represent the earliest signs of brainstem dysfunction, especially if associated with critical alterations of other markers such as ICP or CPP.

From our previous work on comatose patients $^{578}$ and normal subjects ${ }^{18}$ we devised criteria to distinguish changes related to CNS dysfunction from those of non-pathological origin. In this series of patients latency changes fulfilling our quite restrictive criteria did not necessarily make BAEP exceed statistical norms, as established in a control population. In most patients latencies remained within normal limits during the whole episode, and would have not been considered as abnormal had a single BAEP been obtained at that moment. We have previously demonstrated that in patients with irreversible changes progressing up to brain death BAEP abolition mostly occurred before the I-V IPL had exceeded normal values. ${ }^{73}$ This emphasises the fact that during monitoring relative changes from baseline are much more important than comparisons to normative data banks. ${ }^{38}$

In our patients, the significance of transient latency increases was best assessed when studied in correlation with ICP and CPP. Only $50 \%$ of acute ICP/CPP abnormalities were accompanied by an increase of the I-V interval, thus suggesting that not all "significant" pressure changes entail a functional suffering of the brainstem, and that the mere monitoring of ICP values may not always predict their actual incidence on brainstem function. This is consistent with the results of Kawahara et al, who reported that the range of ICP values associated to wave $V$ loss during BAEP monitoring were extremely wide, and could range from 30 to $150 \mathrm{mmHg} .^{11}$ In our patients it was clear that for the same category of pressure changes, the ensuing clinical evolution differed drastically depending on whether a concomitant BAEP modification appeared or not. When ICP changes were accompanied by transient $\mathrm{I}-\mathrm{V}$ increases the subsequent clinical evolution was almost constantly bad, with more than $90 \%$ progression to brain death in the following hours or days, whereas outcome was significantly better (18\% brain death or PVS) in patients whose episodes of pressure change did not entail BAEP modifications.

Transient displacement of the brainstem, either laterally due to uncal herniation or caudally during central herniation, may entail the development of secondary intraparenchymal haemorrhages due to stretching and tearing of perforant branches of the basilar artery. ${ }^{3739} 40$ In dogs submitted to transient ICP rises followed by reperfusion of the perforant arteries, mesencephalic haemorrhages developed due to blood extravasation at the moment of reperfusion ${ }^{41}$ which indicated lesions of brainstem arteries due to the preceding stretching. We suggest that in our patients, transient brainstem distortion secondary to acute ICP rise, and manifested by BAEP changes, might have set up an ongoing and self-perpetuated process, with progressive brainstem deterioration culminating in brain death. This would be consistent with the slow progressive latency increase that followed transient BAEP episodes in several patients (fig 3), and which ultimately lead to brain death in the absence of further ICP/CPP modifications.

At the time our recordings were obtained the detection of transient BAEP changes did not entail a modification of patient's management; therefore we cannot ascertain whether these acute changes are the first signs of a potentially reversible process, or conversely herald an irreversible brainstem deterioration. What seems certain is that BAEP monitoring provides important information on whether a particular episode of ICP rise is affecting 
brainstem function or not. The association of acute ICP rises to BAEP modifications, even if both are transient and totally reversible, is a reliable sign of alarm. In those cases a significant brainstem suffering may be assumed even if pressure modifications do not reach the commonly assumed "critical threshold" values, as BAEP latency changes in that context seem more reliable a warning than absolute intracranial pressure levels.

From this study it may also be concluded that BAEP stability is the only desirable finding during coma monitoring, since any significant modification, even transient, of BAEPs implicates a very poor outcome. It seems reasonable, therefore, to suggest that, in addition to BAEPs, the monitoring of hemispheric function with cortical EPs must be considered to detect the signs preceding irreversible brainstem damage. Technically this can be implemented either by simultaneous monitoring of short- and middle-latency responses (auditory or somatosensory), or by starting monitoring with cortical EPs, and shifting to (or adding) brainstem recordings when the cortical components become altered or disappear.

1 Maresch H, Pfurtscheller G. Simultaneous measurements of auditory brainstem potentials and EEG spectra. Electroencephalography and Clinical Neurophysiology 1983; 56:531-3.

2 Boston JR, Deneault LG. Sensory evoked potentials: a system for clinical testing and patient monitoring. International fournal of Clinical Monitoring and Computing 1984;1:13-19.

3 Maynard DE, Jenkinson JL. The cerebral function analyzing monitor. Anesthesia 1984;39:678-90.

4 Prior PF, Maynard DE. Monitoring cerebral function long term monitoring of EEG and evoked potentials). Amsterdam: term monitoring

5 Bertrand O, Garcia-Larrea L, Artru F, Pernier J, Mauguière F. Brainstem Monitoring I: A system for high rate BAEP sequential monitoring and feature extraction. Electroencephalography and Clinical Neurophysiology 1987;68: 433-45.

6 Pfurtscheller G, Maresch H, Schwarz G, Auer L, González A. Compressed EP and EEG spectra monitoring in the intensive care unit and operating theatre. Electroence-
phalography and Clinical Neurophysiology 1985;61:S27 (Abs).

7 García-Larrea L, Bertrand O, Artru F, Pernier J, Mauguière F. Brainstem monitoring in coma II: Dynamic interpretation of preterminal BAEP changes observed until brain death in deeply comatose patients. Electroencephalography and Clinical Neurophysiology 1987;68:446-57.

8 García-Larrea L, Artru F, Bertrand O, Pernier J, Mauguière F. Transient pharmacological BAEP abolition in coma. Feurology 1988;38:1487-9.

9 Pfurtscheller G, Schwarz G, Schroettner P, et al. Continuous and simultaneous monitoring of EEG spectra and brainstem auditory and somatosensory evoked potentials in the intensive care unit and the operating room. $\mathcal{f}$ Clin Neurophysiol 1987;4:389-96.

10 Buchner H, Ferbert A, Hacke A. Serial recording of median nerve stimulated subcortical somatosensory evoked potentials (SEPs) in developing brain-death. Electroencephalography and Clinical Neurophysiology 1988;69:14-23.

11 Kawahara N, Sasaki Mii K, Tsuzuki M, Takakura K. Sequential changes of auditory brain stem responses in relation to intracranial and cerebral perfusion pressure and initiation of secondary brain stem damage. Acta Neunochirurg 1989;100:142-9.

12 Litscher G, Friehs G, Maresch H, Pfurtscheller G. Electroencephalographic and evoked potentials monitoring in hyperbaric environment. $\mathcal{f}$ Clin Monitoring 1990;6: hyperbari 17.

13 Mauguière F, Garcia-Larrea L, Bertrand $O$. Utility and uncertainties of evoked potential monitoring in the ICU. In: Grundy $L$ and Vitali M, eds. Evoked potentials in ICU and surgical monitoring. Berlin: Springer-Verlag, 1988 153-67.

14 Bertrand O. Système informatisé d'enregistrement séquential des potentiels ézoqués auditifs précoces adapté à la surveillance des malades comateux. PhD Thesis, Lyon (France), 1985.

15 Rougemont J, de Barge $M$, Benavid AL. Un nouveau capteur pour mesure de la pression intracrânienne. Valeu de le dure-mère en tant que membrane susceptible de transmettre les pressions. Neurochirurgie 1971;17: 579-80.

16 Fischer C, Mauguière F, Echallier JF, Courjon J. Contribution of brainstem auditory evoked potentials to diagnosis of tumors and vascular diseases. In: Courjon J, Mauguière F, Revol M, eds. Clinical applications of evoked potentials in neurology. Advances in neurology vol 32. New York:
Raven Press 1982:177-85.

17 Javel E, Mouney DF, McGee JA, Walsh EJ. Auditory brainstem responses during systemic infusion of lidocaine. stem responses during systemic
Arch Otolaryng 1982;108:71-76.

18 Bastuji H, Garcia-Larrea L, Bertrand O, Mauguière F. BAEP latency changes during nocturnal sleep are correlated with body temperature variations, but not with sleep stages. Electroencephalography and Clinical Neurophysiology 1988;70:9-15.

19 Greenberg RP, Becker DP, Miller JD, Mayer DJ. Evaluation of brain function in severe head trauma with multimodality evoked potentials. Pt 2. Localization of brain dysfunction and correlation with postraumatic neurologic conditions. I Neurosurg 1977;47:163-77.

20 Seales DM, RossiterVS, Weinstein ME. Brainstem auditory evoked responses in patients comatose as a result of blunt evoked responses in patients comatose as a
head trauma. $\mathcal{F}$ Trauma 1979;19:347-52.

21 Tsubokawa T, Nishimoto H, Yamamoto T, Kitamura $M$, Katayama Y, Moriyasu N. Assessment of brainstem damage by the auditory brainstem responses in acute severe head injury. I Neurol Neurosurg Psychiatry 1980; 43:1005-11.

22 Uziel A, Benezech J, Lorenzo S, Monstrey Y, Duboin P, Roquefeuil B. Clinical applications of brainstem auditory evoked potentials in comatose patients. In: Courjon J, Mauguière F, Revol M, eds. Clinical Applications of Evoked Potentials in Neurology. Advances in Ner

23 Lütschg J, Pfenninger J, Ludin HP, Vasella F. Brainstem auditory evoked potentials and early somatosensory evoked potentials in neurointensively treated comatose children. Am $\Im$ Dis Children 1983;137:421-6.

24 Brewer CC, Resnick DM. The value of BAEPs in assessment of the comatose patient. In: Nodar, RH, Barber C, ment of the comatose patient. In: Nodar, RH, Barber C,
eds. Evoked potentials II. Boston: Butterworths, 1984: eds. Evo

25 Kaga K, Nagai T, Takamori A. Auditory short-, middle- and long-latency responses in acutely comatose patients. Laryngoscope 1985;95:321-5.

26 Karnaze DS, Weiner JM, Marshall LF. Auditory evoked potentials in coma after closed head-injury. Neurology 1985;35:1122-6.

27 De Weerd AW, Groeneveld C. The use of evoked potentials in the management of patients with severe cerebral trauma. Acta Neurolo Scand 1985;72:489-94.

28 Facco E, Martini A, Zuccarello M, Agnoletto M, Giron GP. Is the auditory brain-stem response (ABR) effective in the Is the auditory brain-stem response (ABR) encephalograassessment of post-traumatic coma? Electroencep

29 Phy and Clinical Neurophysiology 1985;62:332-7. severe head injury by short latency somatosensory and brain-stem auditory evoked potentials. Electroencephalography and Clinical Neurophysiology 1986;65: 188-95.

30 Cenzato M, Ducati A, Fava E, et al. Evaluation of traumatic coma by means of multimodality evoked potentials. In: Grundy L, Vitali M, eds. Evoked potentials. ICU and surgical monitoring. Berlin: Springer-Verlag, 1988: 153-67.

31 Rosenberg C, Starr A. Sensory evoked potentials in coma and brain death. In: Lüders $\mathrm{H}$, ed. Advanced evoked potentials. Boston: Kluwer Academic Publishers, 1989: potentials.

32 Chiappa KH. Evoked potentials in clinical medicine. New York: Raven Press, 1983:145-89.

33 Ferbert A, Buchner H, Brückmann $H$, Zeumer $H$, Hacke W. Evoked potentials in basilar artery thrombosis: correlation with clinical and angiographic findings. Electroencephalography and Clinical Neurophysiology 1988;69: $136-47$.

34 Garcia-Larrea L. Evoked potentials in the comatose patient. In: Osselton J, Binnie C, Prior P, et al, eds. Manual of clinical neurophysiology. London: Butterworths, 1992 (in press).

35 Carlsson CA, von Essen C, Lofgren J. Factors affecting the clinical course of patients with several head injuries. $f$ Neurosurg 1968;29:242-51.

36 Clifton GL, Grossman RG, Makela ME, Miner ME, Handel S, Sadhu V. Neurological course and computerized tomography findings after severe closed head injury. I Neurosurg 1980;52:611-24.

37 Plum F, Posner J. Diagnosis of stupor and coma, 2nd ed. Philadelphia: Davis, 1980.

38 Hacke W. Neuromonitoring. f Neurol 1985;232:125-33.

39 Johnson RT, Yates PO. Clinico-pathological aspects of pressure changes at the tentorium. Acta Radiologica, 1956; 46:242-9.

40 Hassler $O$. Arterial pattern of human brain-stem. Normal appearance and deformation in expanding supratentorial conditions. Neurology 1967;17:368-75.

41 Klintworth KG. Paratentorial growing of human brains with particular reference to transtentorial herniation and the pathogenesis of secondary brainstem hemorrhages. $A m \mathcal{~}$ Path 1968;53:391-9. 